\title{
Symmetric Solutions of a Nonlinear Elliptic Problem with Neumann Boundary Condition
}

\author{
Ana Magnolia Marin Ramirez ${ }^{1}$, Ruben Dario Ortiz Ortiz ${ }^{1}$, Joel Arturo Rodriguez Ceballos ${ }^{2}$ \\ ${ }^{1}$ Group of Waves, Program of Mathematics, Faculty of Exact and Natural Sciences, Cartagena University, \\ Cartagena de Indias, Colombia \\ ${ }^{2}$ Morelia Institute of Technology, Morelia, Mexico \\ Email: amarinr@unicartagena.edu.co,rortizo@unicartagena.edu.co,joel@ifm.umich.mx
}

Received September 3, 2012; revised October 3, 2012; accepted October 11, 2012

\begin{abstract}
We show a result of symmetry for a big class of problems with condition of Neumann on the boundary in the case one dimensional. We use the method of reflection of Alexandrov and we show one application of this method and the maximum principle for elliptic operators in problems with conditions of Neumann. Some results of symmetry for elliptic problems with condition of Neumann on the boundary may be extended to elliptic operators more general than the Laplacian.
\end{abstract}

Keywords: Nonlinear Boundary Value Problems; Elliptic

\section{Introduction}

The maximum principle is one of the most used tools in the study of some differential equations of elliptic type. It is a generalization of the following well-known theorem of the elemental calculus "If $f$ is a function of class $C^{2}$ in $[a, b]$ such that the second derivative is positive on $(a, b)$ then the maximum value of $f$ attains at the ends of $[a, b]$ ”. It is important to point out that the maximum principle gives information about the global behavior of a function over a domain from the information of qualitative character in the boundary and without explicit knowledge of the same function. The maximum principle allows us, for example, to obtain uniqueness of solution of certain problems with conditions of the Dirichlet and Neumann type. Also it allows to obtain a priori estimates for solutions. These reasons make interesting the study of the maximum principle on several forms and its generalizations and the Hopf lemma. For example a geometric version of the maximum principle allows us to compare locally surfaces that coincide at a point. On the other hand, the maximum principle and the Alexandrov reflection principle in [1] have been used to prove symmetries with respect to some point, some plane, symmetries of domain and to determine asymptotic-symmetric behavior of the solutions of some elliptic problems. (See Serrin [2], Gidas, Ni and Nirenberg [3], Gidas, $\mathrm{Ni}$ and Nirenberg [4], Caffarelli, Gidas and Spruck [5], Berestycki and Nirenberg [6]). The first person in use this technic was Serrin. Serrin proved that: "If $u$ is a positive solution of the problem

$$
\Delta u=-1 \quad \text { en } \Omega
$$

which is zero on the boundary and its outer normal derivative on the boundary is constant, then $\Omega$ is a ball and $u$ is radially symmetric with respect to the center of $\Omega$ ”. Using the ideas of Serrin and a version of the maximum principle for functions that do not change of sign, Gidas $\mathrm{Ni}$ and Nirenberg proved that: "If $\Omega$ is a ball, $f \in C^{1}(\mathbb{R})$ and $u$ is a positive solution of the problem,

$$
\Delta u+f(u)=0 \quad \text { on } \quad \Omega
$$

which is zero on the boundary, then $u$ is radially symmetric with respect to the center of the ball". Using the method of reflection and a version of maximum principle for thin domains Berestycki and Nirenberg made a generalization of this statement. Our proof shows that the technic used by Berestycki and Nirenberg for the study of symmetries of solutions of the elliptic problem with Dirichlet condition, can be applied in elliptic problems with Neumann conditions with nonlinear term $f(x, u(x))$.

\section{Maximum Principle and Hopf Lemma}

Our result is based on the well known maximum principle and on the Hopf lemma for the differential operator of the form (see [7-9])

$$
L[u]=a(x) u^{\prime \prime}+b(x) u^{\prime}+c(x) u
$$

where $x$ is in $(a, b)$. We suppose that the coefficients 
$a(x), b(x)$ and $c(x)$ are bounded on $(a, b)$ and $a(x)>0, \quad c(x) \leq 0$ for all $x \in(a, b)$.

\section{Theorem 2.1. (Maximum principle)}

Let $u \in C^{2}((a, b))$ be such that $L[u]>0$. Then $u$ cannot attain its maximum value in $(a, b)$

\section{Lemma 2.2. (Hopf)}

Suppose $u \in C^{2}((a, b)) \cap C^{0}([a, b])$ satisfies

$$
L[u] \geq 0 \text { in }(a, b) \text {. }
$$

Let $x_{0} \in \partial(a, b)$ be such that

- $u$ is continuous at $x_{0}$,

- $u\left(x_{0}\right)>u(x)$ for all $x \in(a, b)$,

- $\frac{\partial u}{\partial \boldsymbol{\eta}}\left(x_{0}\right)$ existe.

Then $\frac{\partial u}{\partial \boldsymbol{\eta}}\left(x_{0}\right)>0$.

\section{Main Result}

Theorem 3.3. Let $u \in C^{2}((-1,1)) \cup C^{0}([-1,1])$ be a solution of

$$
\left\{\begin{array}{l}
a(x) u^{\prime \prime}(x)+b(x) u^{\prime}(x)+c(x) u(x)=f(x, u(x)) \\
\text { on }(-1,1), \\
u^{\prime}(1)=-u^{\prime}(-1),
\end{array}\right.
$$

where $a, c:[-1,1] \rightarrow \mathbb{R}$ are bounded functions and symmetric with respect to the origin such that $a(x)>0$ and $c(x) \leq 0$ for all $x \in[-1,1] ; \quad f \in C^{\prime}\left(\mathbb{R} \times \mathbb{R}^{+}, \mathbb{R}\right)$ is such that $f(x, t)$ is strictly increasing in $t$ for all $x \in[-1,1]$ and is symmetric to $x=0$ for all $t \in \mathbb{R}^{+}$, and $b:[-1,1] \rightarrow \mathbb{R}$ is a bounded function and odd. Then $u$ is symmetric with respect to the origin.

Proof: Define the reflected function of $u$ in $[-1,1]$ by

$$
v(x)=u(-x), \quad x \in[-1,1] .
$$

Hence, $\quad v^{\prime}(x)=-u^{\prime}(-x), v^{\prime \prime}(x)=u^{\prime \prime}(-x)$. Then $\quad v$ satisfies

$$
\left\{\begin{array}{l}
a(x) v^{\prime \prime}(x)+b(x) v^{\prime}(x)+c(x) v(x)=f(x, v(x)) \\
\text { on }(-1,1), \\
v^{\prime}(1)=-v^{\prime}(-1),
\end{array}\right.
$$

Define

$$
w(x)=u(x)-v(x) .
$$

Then $w$ satisfies

$$
\left\{\begin{array}{l}
a(x) w^{\prime \prime}(x)+b(x) w^{\prime}(x)+c(x) w(x) \\
=f(x, u(x))-f(x, v(x)) \\
\text { on } \quad(-1,1) \\
w^{\prime}(-1)=w^{\prime}(1)=0 .
\end{array}\right.
$$

Since $w$ is continuous in $\bar{\Omega}$, there are $x_{M}, x_{m} \in \bar{\Omega}$ such that

$$
w\left(x_{m}\right)=\min _{\bar{\Omega}} w \quad \text { and } \quad w\left(x_{M}\right)=\max _{\bar{\Omega}} w
$$

Suppose that $x_{M}$ or $x_{m} \in(-1,1)$, then if $x_{M} \in(-1,1), w\left(x_{M}\right) \geq 0$ since $w(0)=0$. Further $w^{\prime \prime}\left(x_{M}\right) \leq 0, w^{\prime}\left(x_{M}\right)=0$. Therefore

$$
f\left(x_{M}, u\left(x_{M}\right)\right)-f\left(x_{M}, v\left(x_{M}\right)\right) \leq 0 .
$$

Since $f(., t)$ is strictly increasing in $t$

$$
w\left(x_{M}\right) \leq 0 .
$$

Then

Therefore

$$
w\left(x_{M}\right)=0 .
$$

$$
w(x) \leq 0 \text { for all } x \in[-1,1] .
$$

If $x_{m} \in(-1,1)$, using a similar argue we demonstrate that $w \equiv 0$ on $[-1,1]$ and we obtain the same conclusion. Suppose that $x_{m} \in(-1,1)$, then $w\left(x_{m}\right) \leq 0$. since $w(0)=0$ Further $w^{\prime \prime}\left(x_{m}\right) \geq 0, w^{\prime}\left(x_{m}\right)=0$. Therefore

$$
f\left(x_{m}, u\left(x_{m}\right)\right)-f\left(x_{m}, v\left(x_{m}\right)\right) \geq 0 .
$$

Since $f(., t)$ is strictly increasing in $t$

$$
w\left(x_{m}\right) \geq 0 .
$$

Then

Therefore

$$
w\left(x_{m}\right)=0 .
$$

$$
w(x) \geq 0 \quad \text { for all } \quad x \in[-1,1] .
$$

We conclude

$$
w \equiv 0 \quad \text { on } \quad[-1,1] .
$$

So $u$ is symmetric with respect to the origin.

We will prove that $x_{m}, x_{M}$ do not belong to $\partial[-1,1]$. Suppose now that $x_{m}, x_{M} \in\{-1,1\}$ and $w\left(x_{m}\right)<w(x)<w\left(x_{M}\right)$ for all $x \in(-1,1)$, then $w\left(x_{M}\right)>0$ and $w\left(x_{m}\right)<0$. If $x_{m}=-1$ and $x_{M}=1$, then $w(x) \leq 0$ in $(-1, \alpha)$ and $w(x) \geq 0$ in $(\beta, 1)$, where $\alpha, \beta \in(-1,1)$ are such that $\alpha$ is the first zero of $w$ and $\beta$ is the last. Since $f(., t)$ is strictly increasing in $t$, then

$$
\left\{\begin{array}{l}
a(x) w^{\prime \prime}+b(x) w^{\prime}+c(x) w \leq 0 \quad \text { in } \quad(-1, \alpha), \\
w^{\prime}(-1)=0
\end{array}\right.
$$

and

$$
\left\{\begin{array}{l}
a(x) w^{\prime \prime}+b(x) w^{\prime}+c(x) w \geq 0 \quad \text { in } \quad(\beta, 1), \\
w^{\prime}(1)=0 .
\end{array}\right.
$$


Applying maximum principle and Hopf lemma,

$$
w^{\prime}(-1)>0, \quad w^{\prime}(1)>0,
$$

since $w$ is not constant. Which contradicts the fact that

$$
w^{\prime}(-1)=w^{\prime}(1)=0 .
$$

Hence this case is impossible. It happens equally to $x_{m}=1$ and $x_{M}=-1$. In conclusion we have that $w \equiv 0$ on $[-1,1]$ and therefore $u$ is symmetric with respect to $x=0$.

\section{Example}

Taking

$$
a(x)=1, b(x)=x, c(x)=-1, f(x, u)=x^{2} u
$$

in Theorem 3.3, we have the following system

$$
\left\{\begin{array}{l}
u^{\prime \prime}(x)+x u^{\prime}(x)-u(x)=x^{2} u(x) \\
\text { on }(-1,1), u^{\prime}(1)=-u^{\prime}(-1),
\end{array}\right.
$$

following the steps of the demonstration, it follows that $u$ is symmetric with respect to the origin.

\section{Acknowledgements}

The authors express their deep gratitude to CONACYT México, Programa de Mejoramiento del Profesorado (PROMEP)-México and Universidad de Cartagena for financial support.

\section{REFERENCES}

[1] A. D. Alexandrov, "Uniqueness Theorems for Surfaces in the Large," Vestnik Leningrad University: Mathematics, Vol. 13, No. 19, 1958, pp. 5-8.

[2] J. Serrin, “A Symetry Problem in Potential Theory,” Archive for Rational Mechanics and Analysis, Vol. 43, No. 4, 1971, pp. 304-318. doi:10.1007/BF00250468

[3] B. Gidas, W.-M. Ni and L. Nirenberg, "Symmetry and Related Properties via Maximum Principle,” Communications in Mathematical Physics, Vol. 68, No. 3, 1979, pp. 209-243. doi:10.1007/BF01221125

[4] B. Gidas, W.-M. Ni and L. Nirenberg, "Symmetry of Positive Solutions of Nonlinear Elliptic Equations in $\mathbb{R}^{N}$," In: Mathematical Analysis and Applications, Part A, Academic Press, New York, 1981, pp. 369-402.

[5] L. Cafarelli, B. Gidas and J. Spruck, “Asymptotic Symmetry and Local Behavior of Semilinear Elliptic with Critical Sobolev Growth," Communications on Pure and Applied Mathematics, Vol. 42, No. 3, 1989, pp. 271-297. doi:10.1002/cpa.3160420304

[6] H. Berestycki and L. Nirenberg, "On the Method of Moving Planes and the Sliding Method,” Bulletin of the Brazilian Mathematical Society, Vol. 22, No. 1, 1991, pp. 1-37.

[7] F. John, "Partial Differential Equations," Springer-Verlag, New York, 1982.

[8] M. Protter and H. Weinberger, "Maximum Principle in Differential Equations,” Springer-Verlag, New York, 1984. doi:10.1007/978-1-4612-5282-5

[9] D. Gilbarg and N. Trudinger, "Elliptic Partial Differential Equations of Second Order,” Springer-Verlag, Berlin, Heidelberg, New York, 1977. 